\title{
THE MECHANISM OF DRIVE SYSTEM FLEXIBLE SUSPENSION AND ITS APPLICATION IN LOCOMOTIVES
}

\author{
Yuan Yao, Hong-Jun Zhang, Shi-Hui Luo \\ Traction Power State Key Laboratory, Southwest JiaoTong University, Chengdu, China
}

Submitted 5 March 2012; accepted 20 August 2012; first published online 22 May 2013

\begin{abstract}
The drive system flexibly suspended on the bogie frame is conducive to the lateral dynamic performances of locomotive. In order to clarify the mechanism and optimize the suspension parameters using this model, a bogie dynamics model with 10 degrees of freedom (including the drive system) was established. The lateral dynamic performances were analyzed with the different suspension parameters. The mechanism was determined from the dynamic vibration absorber and the best suspension frequency of the drive system was put forward. The multi-body-dynamics model with two types of drive systems was simulated in locomotives to verify this theoretical analysis. When the suspension frequency is close to the hunting motion frequency of the wheelset, the locomotive dynamics performed the best. The length of the swing rods at the front and rear drive systems were different on the same bogie, which improved the locomotive dynamic performances within a wider range of speed and the wheel conicity. The track shift forces of the locomotive were reduced by $45 \%$ and $34 \%$ respectively with the swing rods on the motor side and the non-motor side relative to the non-flexible suspension.
\end{abstract}

Keywords: locomotive; dynamics; drive system; flexible suspension; lateral stability.

\section{Introduction}

The drive system is suspended flexibly under the bogie frame or under the car body through the swing rods or other elastic. This is a new technology that has been used on high speed trains in recent years (Middendorf 1996; Hödl, Haigermoser 1992; Alfi et al. 2008; Lata 2008). This approach can improve the lateral dynamics performances of locomotives or motorized cars at high running speeds. For example, this technology has been implemented in $160 \mathrm{~km} / \mathrm{h}$ locomotives or high speed motorized cars, such as the CRH3, 'Blue Sword' and the 'China Star' in China. Many $200 \mathrm{~km} / \mathrm{h}$ class locomotives are based on the E120 locomotive in Europe, but their drive systems are changed to flexible suspension (Luo 2005). Alfi et al. (2008) analyzed the effects of motor connection on the critical speed of high-speed railway vehicles. Luo et al. (Luo 2005; Luo, Jin 2007; Luo et al. 2005), Zhang et al. $(2007,2008)$ have made significant contributions to flexible suspension locomotive dynamics and their engineering applications in China. It has long been understood that the mechanism of flexible suspension of the drive system combined with the elastic connection can decouple the mass and inertia between the drive system and bogie frame. This in turn, isolates the lateral movement of the drive system from the bogie frame (Luo 2005; Segieth 1996). This qualitative explanation is not sufficient because the flexible suspension parameters cannot be given out accurately. In this paper, a bogie dynamics model with 10 degrees of freedom, including the elastic connection of drive system, is established. The lateral dynamic performances are analyzed with different suspension parameters. This phenomenon has been made clear from the dynamic vibration absorber and the best suspension frequency is put forward according to the anti-resonance theory. Finally, two types of multi-body-dynamic models of locomotives with different drive system structures were simulated to verify the theoretical analysis. The results provide a theoretical basis for the engineering applications of the drive system suspension optimization parameters of the railway locomotive and power cars.

\section{The Mechanism of Flexible Suspension of Drive System}

\subsection{Dynamics Model of the Flexible Suspension Drive System Bogie}

For a high-speed locomotive, the drive systems are suspended under a bogie frame through a single rubber joint and two swing rods. The single rubber joint and the two swing rods are arranged on the two sides

Corresponding author: Yuan Yao

E-mail: yyuan8848@163.com

Copyright $\odot 2013$ Vilnius Gediminas Technical University (VGTU) Press

http://www.tandfonline.com/TRAN 
of drive systems separately along track direction. The two swing rods can be arranged on the side of the motor or the non-motor end. Relative to the bogie frame, the drive systems possess the lateral and yaw degrees of freedom on the horizontal direction. In this paper, a bogie is equipped Bo' axle arrangement and the impact of car body movement is ignored. The bogie frame is assumed to be connected with the fixed coordinate system through the secondary suspension. The dynamic model is shown in Fig. 1.

Calculating the mass and yaw inertia of the flexible suspended part of drive system, the non-flexible suspended part was added to the bogie frame. Taking the

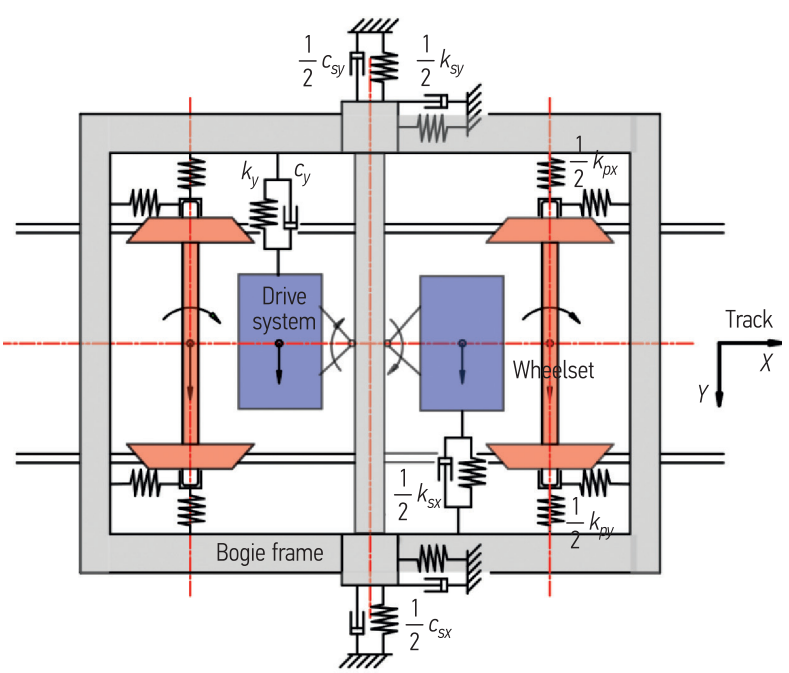

Fig. 1. Dynamics model of the bogie with flexible suspension of drive system lateral and yaw vibrations of the bogie frame, wheelset and drive systems into account, the dynamics model was found to have five rigid bodies and 10 degrees of freedom. The dynamics equation is:

$$
M \ddot{X}+C \dot{X}+K X=Q
$$

where: the $M, C$ and $K$ are the mass, damping and stiffness matrix of the system separately; the $Q$ is the external force vector. The parameters of the dynamics model are shown in Table 1.

For the motion equation of the wheelset, the lateral and yaw movement of wheelset are coupled by the wheel-rail tangential forces. The lateral and yaw frequencies of the wheelset are the same and their phase angles have a certain difference. Ignoring the spin creep forces of the wheelset, the stiffness matrix and damping matrix $K_{w}, C_{w}$ of the linear dynamics equation of the wheelset with elastic positioning in a straight line are as follows:

$$
\begin{aligned}
& K_{w}=\left[\begin{array}{cc}
k_{p y}+k_{g y} & -2 f_{\eta} \\
2 \lambda_{e} l_{0} f_{\xi} / r_{0} & l_{1}^{2} k_{p x}+k_{g \psi}
\end{array}\right] ; \\
& C_{w}=\frac{1}{v}\left[\begin{array}{rr}
2 f_{\eta} & \\
& 2 l_{0}^{2} f_{\xi}
\end{array}\right] .
\end{aligned}
$$

For the asymmetric stiffness matrix $K_{w}$, there are complex eigenvalues for the corresponding system matrix. If any real part of the eigenvalues is positive, the system is unstable. For the wheel-rail system, the non-diagonal items in $K_{w}$ represent the coupling of the wheelset's lateral and yaw movement, which input energy into the system while the $C_{w}$ consumes energy. From the above

\begin{tabular}{|c|c|c|c|c|c|}
\hline Symbol & Physics mean & Value & Symbol & Physics mean & Value \\
\hline$m_{w}$ & Mass of wheelset & $3085 \mathrm{~kg}$ & $2 b$ & Wheel base & $2.5 \mathrm{~m}$ \\
\hline$I_{w}$ & Yaw inertia of wheelset & $2024 \mathrm{~kg} \cdot \mathrm{m}^{2}$ & $2 l_{0}$ & Distance of contact spot & $1493 \mathrm{~mm}$ \\
\hline$m_{f}$ & $\begin{array}{l}\text { Mass of bogie (include non-flexible } \\
\text { suspend part of drive system) }\end{array}$ & $7186 \mathrm{~kg}$ & $2 l_{1}$ & $\begin{array}{l}\text { Lateral spacing of } \\
\text { primary } \\
\text { suspend }\end{array}$ & $2200 \mathrm{~mm}$ \\
\hline$I_{f}$ & $\begin{array}{l}\text { Yaw inertia of bogie (include } \\
\text { non-flexible suspend part) }\end{array}$ & $9751 \mathrm{~kg} \cdot \mathrm{m}^{2}$ & $2 l_{2}$ & $\begin{array}{c}\text { Lateral spacing of } \\
\text { secondary } \\
\text { suspend }\end{array}$ & $2200 \mathrm{~mm}$ \\
\hline$m_{d}$ & $\begin{array}{l}\text { Mass of flexible suspend part of drive } \\
\text { system }\end{array}$ & $1765 \mathrm{~kg}$ & $r_{0}$ & Wheel rolling radius & $0.625 \mathrm{~m}$ \\
\hline$I_{d}$ & Yaw inertia of flexible suspend part & $1019 \mathrm{~kg} \cdot \mathrm{m}^{2}$ & $\lambda_{e}$ & $\begin{array}{l}\text { Wheel-rail contact } \\
\text { conicity }\end{array}$ & 0.15 \\
\hline$k_{p x}$ & Primary longitudinal stiffness per axle & $40 \mathrm{kN} / \mathrm{mm}$ & $f_{\xi}$ & $\begin{array}{l}\text { Longitudinal creep } \\
\text { coefficient }\end{array}$ & $8.624 \mathrm{e} 6$ \\
\hline$k_{p y}$ & Primary lateral stiffness per axle & $6 \mathrm{kN} / \mathrm{mm}$ & $f_{\eta}$ & Lateral creep coefficient & $8.144 \mathrm{e} 6$ \\
\hline$k_{s x}$ & Secondary longitudinal stiffness & $0.8 \mathrm{kN} / \mathrm{mm}$ & $k_{g y}$ & Gravitational Stiffness & $41.4 \mathrm{kN} / \mathrm{m}$ \\
\hline$k_{s y}$ & Secondary lateral stiffness & $0.8 \mathrm{kN} / \mathrm{mm}$ & $k_{g \psi}$ & $\begin{array}{c}\text { Gravitational angular } \\
\text { stiffness }\end{array}$ & $-23 \mathrm{kN} / \mathrm{rad}$ \\
\hline$c_{s x}$ & Secondary longitudinal damping & $1000 \mathrm{kN} \cdot \mathrm{s} / \mathrm{m}$ & $k_{r}$ & $\begin{array}{l}\text { Contact stiffness of } \\
\text { wheel-rail }\end{array}$ & $50 \mathrm{kN} / \mathrm{mm}$ \\
\hline$c_{s y}$ & Secondary lateral damping & $60 \mathrm{kN} \cdot \mathrm{s} / \mathrm{m}$ & $v$ & Speed & $-\mathrm{m} / \mathrm{s}$ \\
\hline
\end{tabular}
equation, $C_{w}$ is related to the speed $v$. As $v$ increases, the

Table 1. The parameters of model 
damping decreases. In a period of hunting motion, if the input and the consumed energy are equal, the linear system is in the critical stability state. The corresponding speed $v$ is the linear critical speed.

The lateral and yaw vibration of drive system have the same frequency and attenuation coefficient. The expression of lateral stiffness $k_{y}$ and damping $c_{y}$ of the drive system suspension are defined as follows:

$$
\begin{aligned}
& k_{y}=\left(2 \pi f_{y}\right)^{2} m_{d} ; \\
& c_{y}=\xi_{y} 2 m_{d}\left(2 \pi f_{y}\right),
\end{aligned}
$$

where: $f_{y}$ is the drive system lateral suspension frequency; $\xi_{y}$ is the drive system lateral suspension-damping ratio; $m_{d}$ is the mass of flexible suspended parts of drive system. Similarly, we can get the expression of yaw stiffness and damping between the drive system and bogie frame.

\subsection{Effect of Flexible Suspension on the Linear Stability of the Bogie}

For the rigid wheelset on the rail vehicle, the wheelsets represent the hunting motion on the track because of the conicity of the wheel profile. As the primary guide stiffness and the gravity, the hunting motion of the wheelset is stable within a certain range of speeds. That is, the wheelset tends to the center of the track. When the vehicle speed exceeds a certain value (called critical speed), the maximal lateral displacement of the wheelset tends towards a nonzero value (the wheel-rail gap usually). In engineering, when the lateral displacement of the wheelset can't converge quickly, it known as 'vehicle lateral instability'. Due to the changes of some parameters, such as the increases in the wheel conicity caused by wear, the vehicle will reduce the lateral stability significantly. The lateral stability will worsen the vehicle dynamic performances or even cause derailment. Thus, the rail vehicle requires the lateral stability to be routinely checked to ensure a high safety margin. The lateral stability of the rail vehicle has been one of the important and difficult problems in vehicle system dynamics (Sun 1993).

Checking the lateral stability of the vehicle includes both linear and nonlinear stability. For the linear stability, you can estimate the vehicle's critical speed and optimize some parameters quickly, while identifying the instability mode through the rigid body vibration frequencies and the corresponding mode shapes.

The eigenvalues and eigenvalue vectors of the homogeneous items of equation (1) can be calculated in the way of state space for the non-symmetrical stiffness and non-proportional damping matrix. The imaginary and real part of each eigenvalue denote the circular frequency of this vibration and its attenuation coefficient. The eigenvalue vector denotes the rigid mode shapes of the corresponding frequency. The root locus curves of the linear system are composed of all eigenvalues at all calculated speeds. If the real part of all eigenvalues are negative, the motion of the system is stable.

In order to discuss the effect of the structure form of drive systems and the suspension parameters on the bogie lateral stability, the linear stability of 4 types of models categorized according to 6 conditions and analyzed:

1. no drive system model;

2. drive system fixed to the bogie frame non-flexibly;

3. single wheelset with elastic positioning, that when equivalent to the bogie frame has only one degree of freedom with forward direction;

4. drive system flexible suspension, $f_{y}=3 \mathrm{~Hz}, \xi_{y}=1.0$;

5. drive system flexible suspension, $f_{y}=3 \mathrm{~Hz}, \xi_{y}=0.3$;

6. drive system flexible suspension, $f_{y}=4 \mathrm{~Hz}, \xi_{y}=0.3$.

Fig. 2 shows the calculated linear critical speeds of the different models and suspension parameters of the drive systems. The lateral stability of the drive system is worst when fixed to the bogie frame non-flexibly because the drive system increases the mass and yaw inertia of the bogie frame. The single wheelset with flexible positioning means the bogie frame does not participate in hunting motion and its linear critical speed is the largest. Fig. 3 shows the root locus curves of the three models. For the bogie model, the smaller imaginary part are the frequencies of the wheelset hunting $(1 \div 4 \mathrm{~Hz})$ and the bigger are the lateral vibration frequencies of the bogie frame $(5 \div 10 \mathrm{~Hz})$. The critical speeds of the bogie

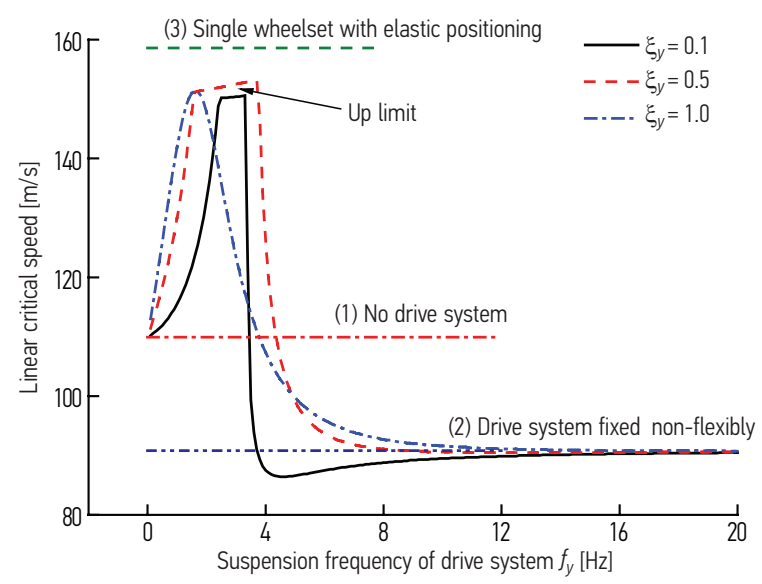

Fig. 2. The linear critical speed

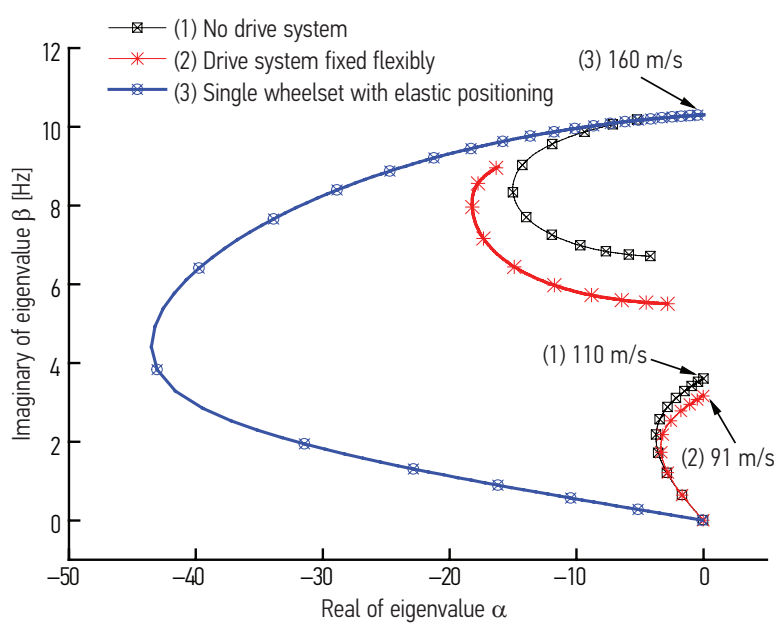

Fig. 3. The root locus curves 
with the flexible suspension are related to the suspension frequency $f_{y}$. When the $f_{y}$ is 0 , it is equivalent to no drive system. The large $f_{y}$ means that the drive system is fixed to the bogie frame non-flexibly. When $f_{y}$ is close to the hunting frequency of wheelset, the lateral stability of the bogie with drive system flexible suspension is significantly better than no drive system and the critical speed is related to suspension dumping $\xi_{y}$. The flexible suspension of the drive system is equivalent to the dynamic vibration absorber and its lateral and yaw vibrations absorb the vibration of the bogie frame. When $f_{y}$ is close to the excitation frequency, the vibration amplitude of the bogie frame is at it's minimum and that enhances the lateral stability of the bogie. In Fig. 4, the corresponding lateral displacement of the drive system is at it's lowest when the critical bogie speed is at it's maximum, rather than the relative displacement between drive system and bogie frame (interpreted as dissipating energy of suspension damping). Thus, this exercise verifies the explanation of the dynamic vibration absorber.

The first instable mode of rail vehicles is usually the bogie hunting motion, that is the frequencies and phases of the wheelsets and frame motions are the same. Except for the curve (5) in Figs 3 and 5, the first instable mode is bogie hunting as the speed increases. The first instable mode of curve (5) is the lateral vibration of the bogie frame. The critical speed of the bogie with drive system flexible suspension are the upper limits of curves in the Fig. 2. If the instability of the rail vehicle occurs,

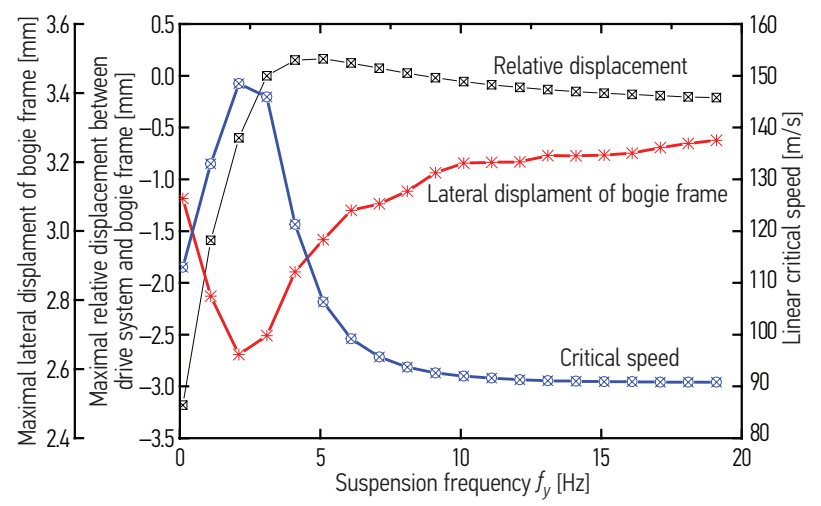

Fig. 4. The simulation results

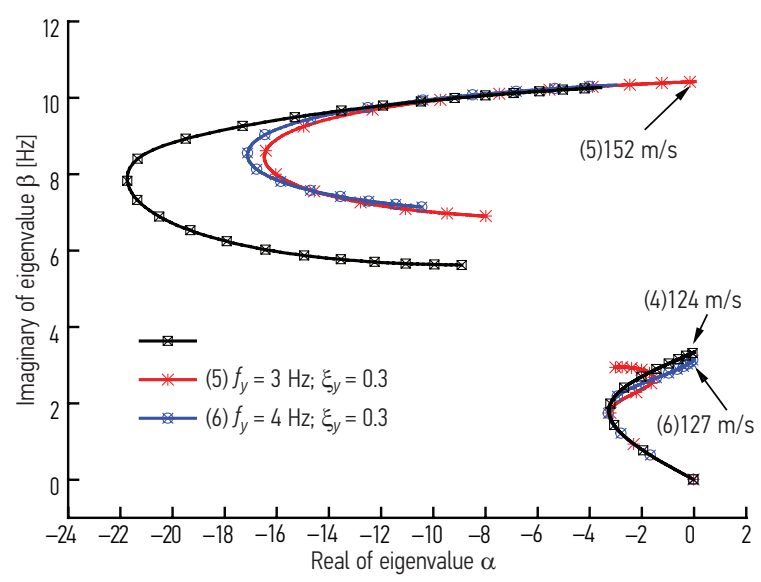

Fig. 5. The root locus curves the main vibration frequencies of all rigid bodies in the vehicle is the unstable mode frequency. The corresponding lateral vibration frequency of the bogie frame is high and can lead to a severe vibration. Thus, the suspension parameters of the vehicle should be first be optimized to avoid bogie frame lateral instability.

\subsection{Effect of Flexible Suspension on the Track Shift Forces}

There is a large dynamic force between the wheel and rail due to the track irregularity. The track shift forces (sum of the guiding forces on the two wheels of the same axle) is an important indicator of a vehicle's dynamic performance. As the track shift forces increase with the increasing running speed, the high-speed vehicle attention is affected by the low dynamic action force. In order to study the effects of flexible suspension on the track shift forces, the dynamic model of the bogie in equation (1) which takes the horizontal track irregularity and the wheel-rail gap into account was simulated, and the flange contact are used the unilateral contact stiffness $k_{r}$ and damping to simulate. The numerical method of Runge-Kutta is used to get numerical results and statistically evaluate the maximal track shift forces.

The simulation results of different suspension frequencies $f_{y}$ on the AAR5 class track irregularity are shown in Figs 6 and 7. The effect of flexible suspension

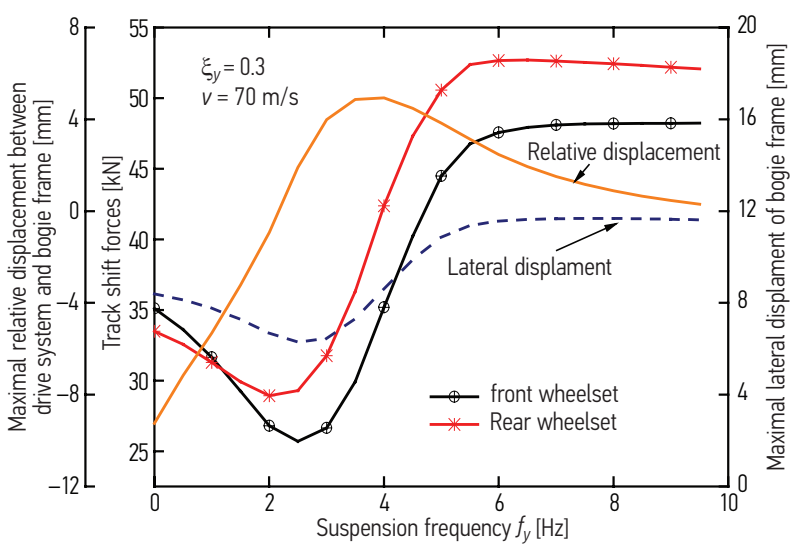

Fig. 6. The simulate results with different $f_{y}$

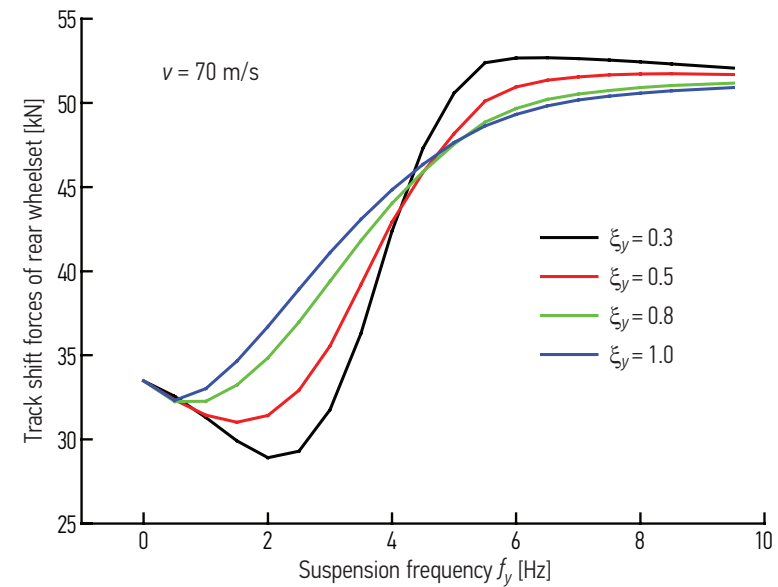

Fig. 7. The effect of $\xi_{y}$ on the track shift forces 
on track shift forces are the same as the lateral stability. The flexible suspension of drive system reduces the track shift forces and the lateral and yaw displacement of the bogie frame. The track shift forces and the bogie frame displacement are lowest when the suspension frequency is close to the hunting frequency. In Fig. 7, when the range of hunting frequency is small, a smaller suspension dumping $\xi_{y}$ is beneficial to reduce the track shift forces.

The rear wheelset of the track shift force is usually bigger than the front wheelset on a straight line. The simulation results show that the suspension frequency $f_{y}$ has influences on the ratio of the track shift forces of the rear and front wheelsets. The bogie lateral acceleration on the front and rear wheelsets is shown as follows respectively in the paper by Alfi et al. (2008):

$$
\begin{aligned}
& \ddot{y}_{1}=\ddot{y}_{f}+p \ddot{\psi}_{f}=-\omega^{2}\left(y_{0}+p \psi_{0}\right) e^{i \omega t} ; \\
& \ddot{y}_{2}=\ddot{y}_{f}-p \ddot{\psi}_{f}=-\omega^{2}\left(y_{0}-p \psi_{0}\right) e^{i \omega t} .
\end{aligned}
$$

The bogie lateral acceleration on the front and rear wheelsets is the vector sum of the lateral and yaw accelerations of the bogie. The acceleration vector is shown in Fig. 8. The analysis of the linear vehicle system shows that the vibration frequency and phase of the wheelsets and bogie frame in one bogie (caused by the wheelset hunting motion) are the same, and the yaw phase angle of the bogie frame is about 90 degrees from the lateral vibration of bogie frame. As shown in Fig. 8, when the phase difference is 90 degrees, the lateral acceleration of the front and rear wheelsets on the bogie are equal.
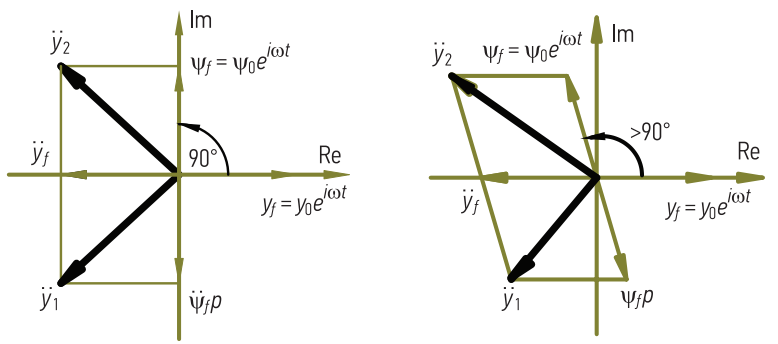

Fig. 8. The vector relationship of acceleration

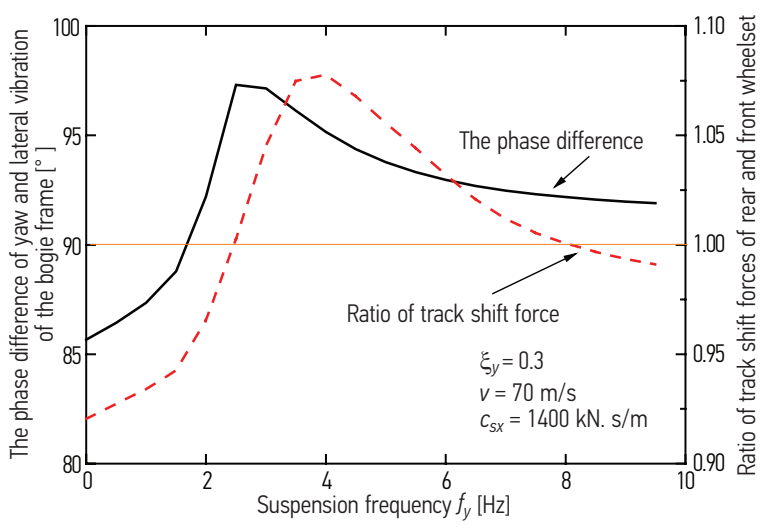

Fig. 9. The effect of $f_{y}$ on the ratio of track shift forces and phase difference
When the phase difference is greater than 90 degrees, the lateral acceleration of the rear wheelset position on bogie is bigger than the front wheelset position. These are the intuitionistic explanations to explain the different track shift forces of the front and rear wheelsets in one bogie on a straight line.

The phase difference of the yaw and lateral vibrations of the bogie frame is related to the mass and yaw inertia and the horizontal suspension parameters of the bogie frame. The different suspension parameters of drive systems can affect the bogie frame's natural frequency of lateral vibration and the phase difference. Fig. 9 shows the effect of the suspension frequency on the ratio of track shift forces. The simulation results show that although the relationship of the phase difference and the ratio of track shift forces is not in strict accordance with the vector analysis in Fig. 8, the overall trend is roughly consistent. The smaller lateral suspension stiffness of the drive system is beneficial to balance the track shift forces of the front and rear wheelsets.

\section{The Theoretical Explanation of the Flexible Suspension}

For the locomotive with flexible suspension, when the suspension frequency of drive system is close to the wheelset hunting frequency, the locomotive will have the best dynamic performance. In order to explain this phenomenon, the simplified model of the bogie dynamic vibration absorber is established, using the anti-resonance theory to explain the mechanism and put forward the optimal value of the suspension parameter of flexible suspension. For the simplified model as shown in Fig. 10, the system's DOFs are equivalent lateral movements, and the dynamics parameters are equivalent parameters. Assuming the lateral position of car body is fixed, this model takes into account the lateral vibration degrees of freedom of the bogie frame and drive system. The horizontal simple harmonic motion of the wheelsets serves as the system's excitation.

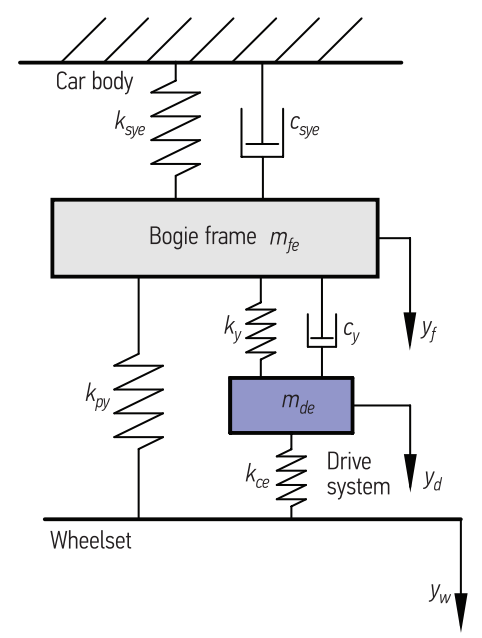

Fig. 10. The lateral simplified model of the railway vehicle 
Ignoring the damping, the transfer function of the ratio of lateral amplitude of the bogie frame $y_{f}$ and the wheelset $y_{w}$ is as follow:

$$
\begin{aligned}
& G_{11}(\omega)=\left|\frac{y_{f}}{y_{w}}\right|= \\
& \frac{k_{p y}\left(k_{y}+k_{c e}-m_{d e} \omega^{2}\right)+k_{c e} k_{y}}{\left(k_{s y e}+k_{p y}+k_{y}-m_{f e} \omega^{2}\right)\left(k_{y}+k_{c e}-m_{d e} \omega^{2}\right)-k_{y} k_{y}},
\end{aligned}
$$

where: $k_{p y}$ is the primary lateral stiffness; $k_{c e}$ is the equivalent combination lateral stiffness of the transmission of double hollow shaft; $k_{\text {sye }}$ is the equivalent secondary lateral stiffness; $m_{f e}$ is the equivalent mass of the bogie frame; $m_{d e}$ is the equivalent mass of flexible suspended parts of the drive system; $\omega$ is the excitation frequency. When the system parameters and $\omega$ are certain, the ratio of vibration amplitudes of the bogie frame and the wheelset is a constant. Equation 7 equals 0 when $\omega=\omega_{n}$ satisfies the following:

$$
k_{p y}\left(k_{y}+k_{c e}-m_{d e} \omega_{n}^{2}\right)+k_{c e} k_{y}=0,
$$

when the bogie frame does not move, it is known as anti-resonance. The corresponding excitation frequency $\omega_{n}$ is known as the 'anti-resonance frequency'. Antiresonance frequency is different from the resonant frequency. When the denominator of $G_{11}(\omega)$ is zero, the corresponding excitation frequency is the resonant frequency, while the anti-resonance frequency is when the numerator is zero. For the vibration system with $n$ degrees of freedom, there are $n$ resonance frequencies and $n$ anti-resonance frequencies, respectively (Sun 1993).

The hunting frequency is the main frequency within the lower frequency region of the lateral wheelset movement. When the speed is constant, the excitation frequency $\omega_{n}$ is the hunting frequency $\omega_{0}$. So the optimal lateral suspension stiffness $k_{y}$ of drive system is as follow:

$$
k_{y}=\frac{k_{p y}}{k_{p y}+k_{c e}}\left(m_{d e} \omega_{o}^{2}-k_{c e}\right) .
$$

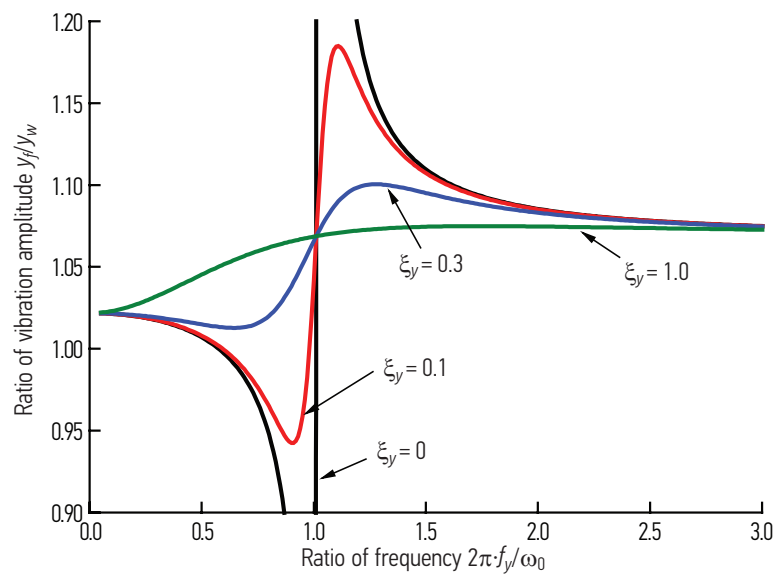

Fig. 11. The vibration amplitude ratio of the bogie frame and wheel-set with different $f_{y}$
From the above equation, the corresponding $k_{y}$ increases as the $\omega_{0}$ and $m_{d e}$ increase and the $k_{y}$ decreases as $k_{c e}$ increases. As the $k_{c e}$ becomes smaller than the $k_{p y}$ (Zhang et al. 2008), we conclude that:

$$
k_{y} \approx m_{d e} \omega_{0}^{2}-k_{c e} .
$$

As the hunting frequency of wheelset is relevant to the running speed, a reasonable value of $k_{y}$ can be selected based on the common speed. To weaken the lateral vibration and reduce the resonance amplitude of drive system, the drive system is connected with the lateral damper. Fig. 11 shows the vibration amplitude ratio between the bogie frame and wheelset with the different $\xi_{y}$. The horizontal coordinate is the ratio of suspension frequency of the drive system and the wheelset hunting frequency. As the effect of suspension dampens, when the ratio of suspension frequency and hunting frequency is slightly less than 1 , the vibration amplitude ratio of the bogie frame and wheelset is lowest.

\section{The Application of Different Suspension Frequencies on the Same Bogie}

There is no optimal suspension frequency of the drive system for all conditions due to changes in hunting frequencies with the changes in running speeds and wheel conicity. The hunting frequencies of the wheelset usually increase with the increase of the running speeds and the wheel conicity $\lambda_{e}$ as shown in Fig. 12. From the mechanism of flexible suspension, the suspension frequencies of the drive system should be close to the hunting frequency to support better dynamic performances of the rail vehicle. The suspension frequencies cannot be changed for the mechanical connection without a control system. Thus, the application of different suspension frequencies of the drive system on the same bogie is an effective problem-solving measure.

Figs 13-15 show the simulation results of the bogie model shown in Fig. 1 with different suspension frequencies and different wheel conicity. The $3 \mathrm{~Hz}$ suspension frequency of the front and rear drive system reduces the track shift forces when the bogie runs at high speed, and

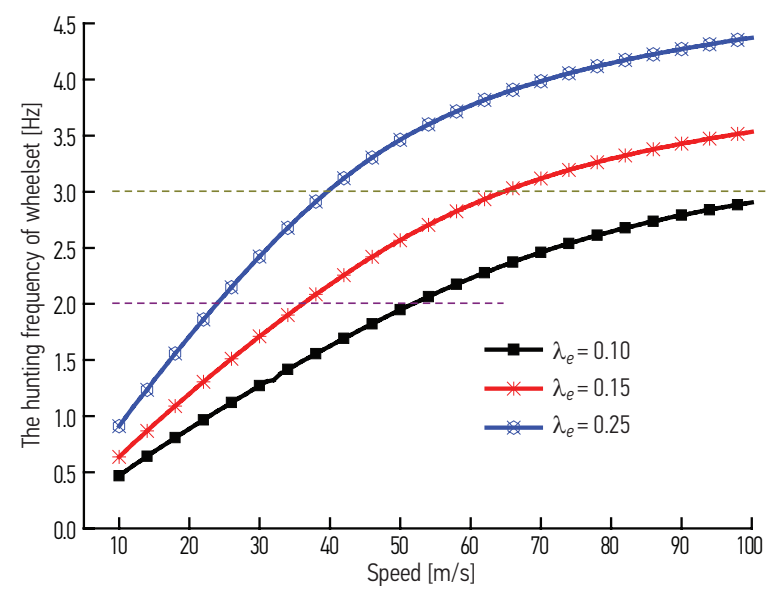

Fig. 12. The hunting frequencies of wheelsets with the different speed and wheel conicities $\lambda_{e}$ 


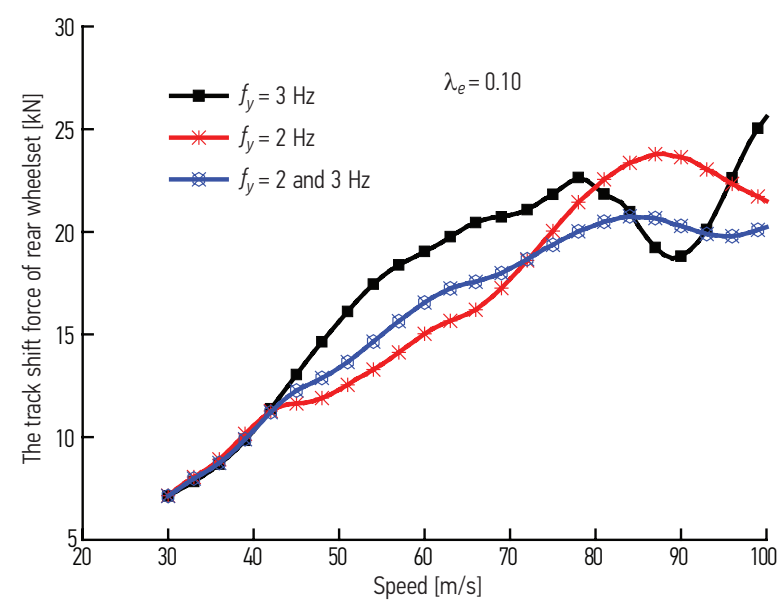

Fig. 13. The track shift forces with the different suspension frequencies at $\lambda_{e}$ is 0.1

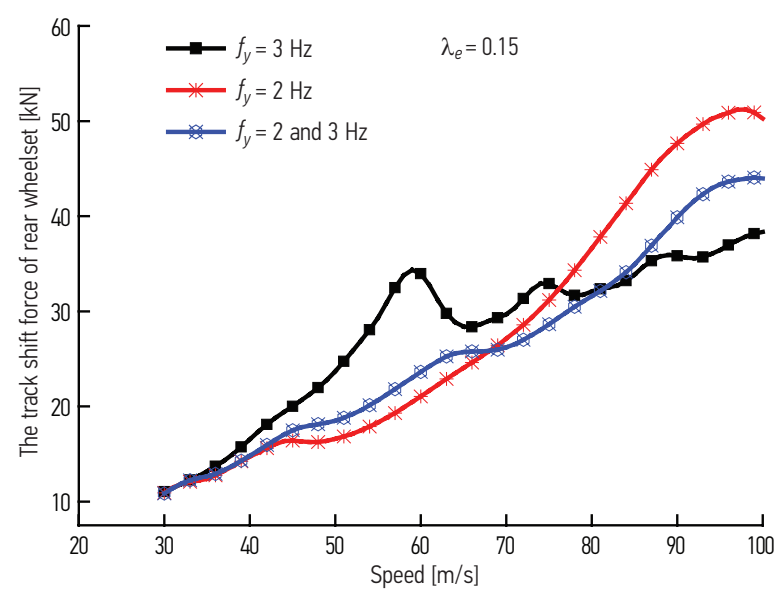

Fig. 14. The track shift forces with the different suspension frequencies at $\lambda_{e}$ is 0.15

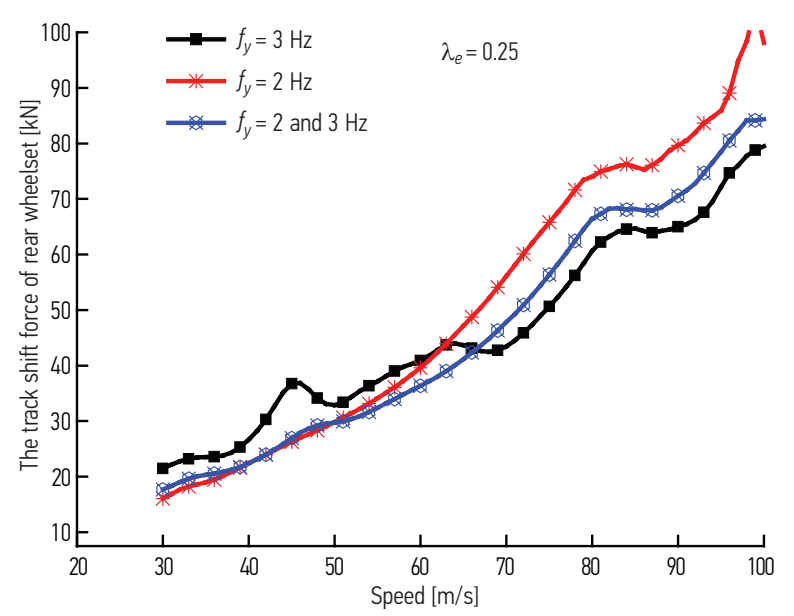

Fig. 15. The track shift forces with the different suspension frequencies at $\lambda_{e}$ is 0.25

the $2 \mathrm{~Hz}$ suspension frequency reduces the track shift forces at lower speed. The suspension frequencies are 2 and $3 \mathrm{~Hz}$ of the front and rear drive system respectively and can improve the dynamic performances for a wide range of speeds. The appropriate running speeds for the flexible suspension decrease with an increase in wheel conicity.

\section{The Suspension Frequencies of Two Types of Drive Systems}

For a high-speed locomotive, the drive systems are suspended under a bogie frame through a single rubber joint and two swing rods. The single rubber joint and the two swing rods are arranged on the two sides of the drive systems separately along the track direction. The swing rods can be arranged close either to the motor, or to the other side, as shown in Figs 16 and 17 respectively. The wheelset and the drive system are connected by the transmission of double hollow shafts. The combined lateral stiffness is $k_{c}$ as shown in the figures. A lateral damper shown as lateral damping $c_{y}$ in the figures is arranged between the drive system and the bogie frame. The positions of the mentioned components above are shown in Figs 16 and 17.
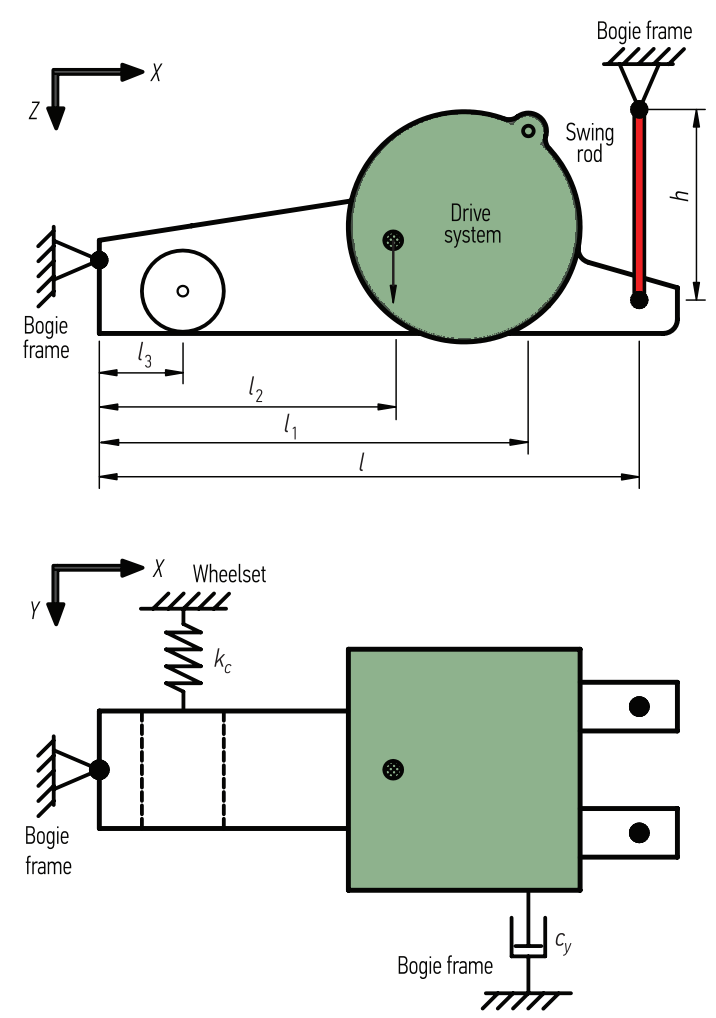

Fig. 16. The flexible suspension drive system $A$

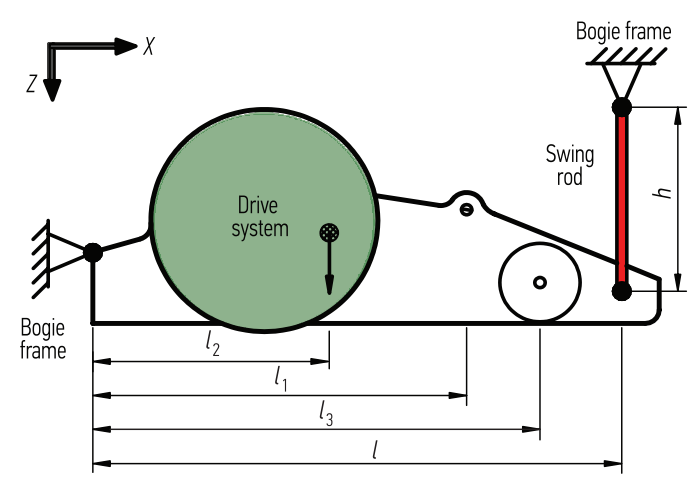

Fig. 17. The flexible suspension drive system $B$ 
The lateral natural frequency of the drive system is defined as the suspension frequency, which depends on the length of the swing rods $h$, the $k_{c}$ and the corresponding positions. Assuming that the bogie frame and wheelset are fixed and the angular displacement of the swing $\theta$ is small, the horizontal motion equation for the drive system is shown as:

$$
\begin{aligned}
& \left(m_{d} i_{2}^{2} h^{2}+I_{d} h^{2} / l^{2}\right) \ddot{\theta}+c_{y} i_{1}^{2} h^{2} \dot{\theta}+ \\
& \left(m_{d} g i_{2} h+k_{c} i_{3}^{2} h^{2}\right) \theta=0 .
\end{aligned}
$$

The parameters of $m_{d}$ and $I_{d}$ are the mass of the drive system and the yaw inertia respectively. The $g$ is the gravity acceleration. The ratio of the corresponding position parameters to $l$ is $i_{k}=l_{k} / l, k=1,2,3$. follows:

The suspension frequency of the drive system is as

$$
f_{y}=\frac{1}{2 \pi} \sqrt{\frac{m_{d} g i_{2} h+k_{c} i_{3}^{2} h^{2}}{m_{d} i_{2}^{2} h^{2}+I_{d} h^{2} / l^{2}}} \approx \frac{1}{2 \pi} \sqrt{\frac{l g}{l_{2} h}+\frac{k_{c}}{m_{d}}\left(\frac{l_{3}}{l_{2}}\right)^{2}} .
$$

The $f_{y}$ decreases when the $h$ and $l_{2}$ increase and the $k_{c}$ and $l_{3}$ decrease. Namely the connecting lateral stiffness between the drive system and the frame decreases.

This paper selected two different locomotives in the $200 \mathrm{~km} / \mathrm{h}$ speed class as the analysis objects, of which drive systems were flexibly suspended on the frame respectively in the two structural forms mentioned above. The structural parameters of the two drive systems are shown in Table 2.

Table 2. The parameters of the drive system

\begin{tabular}{lccccc}
\hline Parameters & $h[\mathrm{~m}]$ & $l[\mathrm{~m}]$ & $l_{1}[\mathrm{~m}]$ & $l_{2}[\mathrm{~m}]$ & $l_{3}[\mathrm{~m}]$ \\
\hline $\begin{array}{l}\text { Drive } \\
\text { system } A\end{array}$ & 0.47 & 1.7655 & 1.384 & 1.078 & 0.612 \\
\hline $\begin{array}{l}\text { Drive } \\
\text { system } B\end{array}$ & 0.485 & 1.812 & 1.622 & 0.808 & 1.2 \\
\hline \hline Parameters & $k_{c}[\mathrm{kN} / \mathrm{mm}]$ & $c_{y}[\mathrm{kN} \cdot \mathrm{s} / \mathrm{m}]$ & $m_{d}[\mathrm{~kg}]$ & $I_{d}\left[\mathrm{~kg} \cdot \mathrm{m}^{2}\right]$ \\
\hline $\begin{array}{l}\text { Drive } \\
\text { system } A\end{array}$ & 0.34 & 40 & 4000 & 1070 \\
\hline $\begin{array}{l}\text { Drive } \\
\text { system } B\end{array}$ & 0.34 & 40 & 4146 & 683 \\
\hline
\end{tabular}

According to Equation 7 , the $f_{y}$ reduces with increasing $h$ and decreasing $k_{c}$. The suspension frequencies of two types of drive system are shown in Figs 18 and 19. The shorter length of the swing rod has a greater impact on $f_{y}$. When the $h$ is greater than $100 \mathrm{~mm}$, the $f_{y}$ is less than $3 \mathrm{~Hz}$. It's not obvious if one is able to reduce the lateral stiffness of the drive system by continuing to increase the length of the swing rod. On the drive system $B$, because the swing rod and the transmission of the double hollow shafts are on the same side, the $k_{c}$ has an obvious impact on the $f_{y}$ and the $f_{y}$ is larger. When the hunting frequency of the wheelset is $2 \mathrm{~Hz}$, for the $k_{c}$ in Table 1, the optimal length of the swing rod on drive system $A$ is $0.1 \mathrm{~m}$, while the longer length of the

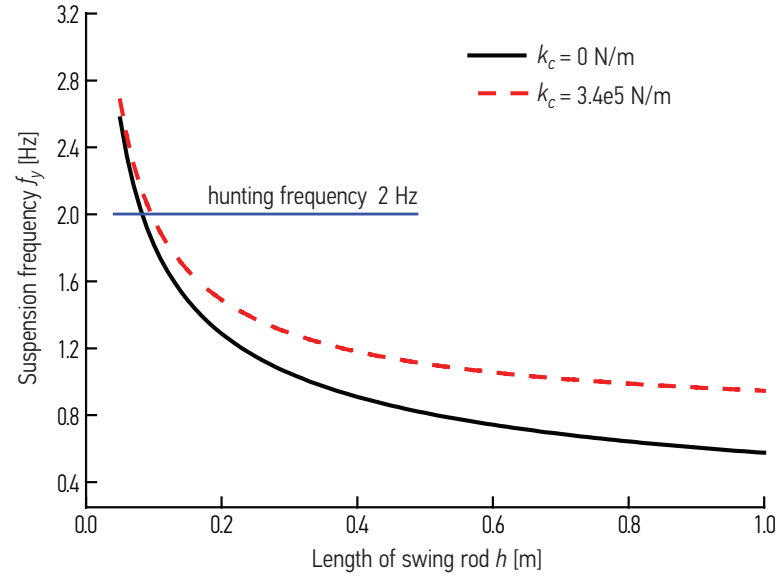

Fig. 18. The suspension frequency of drive system $A$

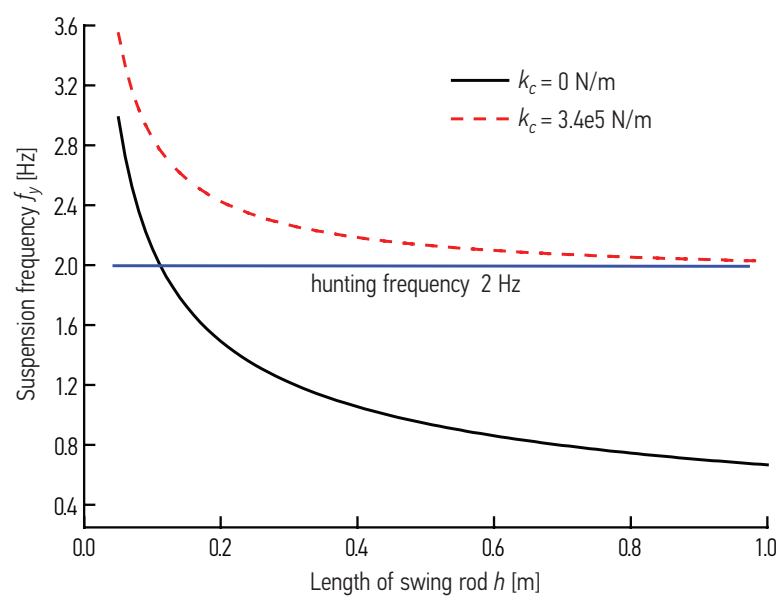

Fig. 19. The suspension frequency of drive system $B$

swing rod on the drive system $B$ is better in the calculation range. But the optimal length is $0.1 \mathrm{~m}$ if the $k_{c}$ equals $0 \mathrm{~m}$.

\section{Vehicle Dynamics Simulation and Verification}

The dynamics analyses were carried out on two different locomotives in order to verify the effect of drive system flexible suspension on the vehicle system dynamics. The two locomotives dynamics models were established by the multi-body-dynamics software SIMPACK, which is shown in Fig. 20 and the main parameters are given in Table 3 . The drive systems $A$ and $B$ were applied to the locomotives 1 and 2 with the standard gauge respectively. The wheel profile was of the wear type JM3. The track irregularity of DB high class are adopted in a straight line for simulation, in which the speed is $200 \mathrm{~km} / \mathrm{h}$ and the corresponding hunting frequency of the wheelset is $2 \mathrm{~Hz}$.

The track shift force curves with different swing rod lengths are shown in Figs 21 and 22 for locomotive 1. The track shift force of the rear wheelset is greater than that of the front wheelset in one bogie. The maximum displacements of the bogie and the track shift forces have the same rules with the changing swing rod. The corresponding minimum track shift forces and lateral displacement appear when the length of the swing rod is $0.1 \mathrm{~m}$ for the 
Table 3. The main parameters of locomotives

\begin{tabular}{|c|c|c|c|c|c|}
\hline Parameters & Axle arrangement & Axle load $[\mathrm{t}]$ & Motor arrangement & Wheel base $[\mathrm{m}]$ & Bogie base $[\mathrm{m}]$ \\
\hline Locomotive 1 & $2 C_{\mathrm{O}}$ & 21 & Sequence & $2.0 \div 2.0$ & 13.05 \\
\hline Locomotive 2 & $2 C_{\mathrm{O}}$ & 21 & Sequence & $2.15 \div 2.0$ & 11.76 \\
\hline Parameters & \multicolumn{2}{|c|}{$\begin{array}{l}\text { Primary longitudinal stiffness } \\
{[\mathrm{kN} / \mathrm{mm}]}\end{array}$} & \multicolumn{2}{|c|}{$\begin{array}{c}\text { Primary lateral stiffness } \\
{[\mathrm{kN} / \mathrm{mm}]}\end{array}$} & $\begin{array}{c}\text { Second lateral stiffness } \\
{[\mathrm{kN} / \mathrm{mm}]}\end{array}$ \\
\hline Locomotive 1 & \multicolumn{2}{|c|}{28.67} & \multicolumn{2}{|c|}{3.67} & 0.428 \\
\hline Locomotive 2 & \multicolumn{2}{|c|}{28.7} & \multicolumn{2}{|c|}{3.74} & 0.426 \\
\hline
\end{tabular}

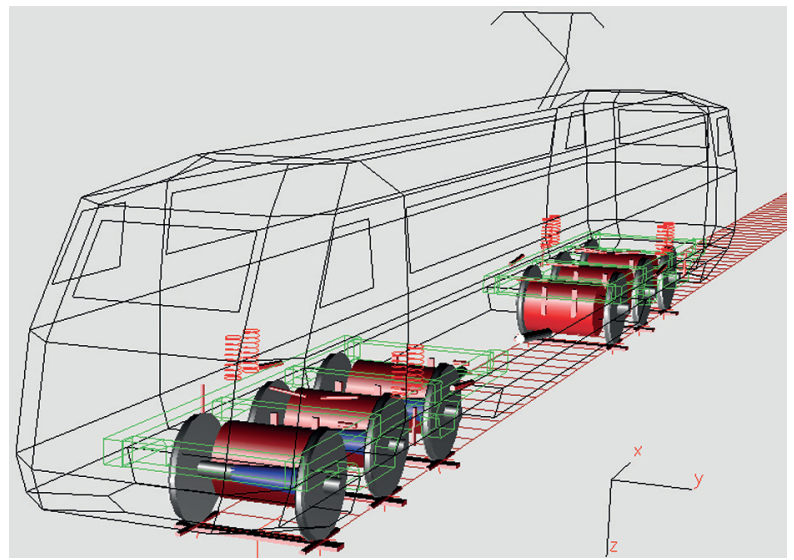

Fig. 20. The multiple-body-dynamics model of locomotive

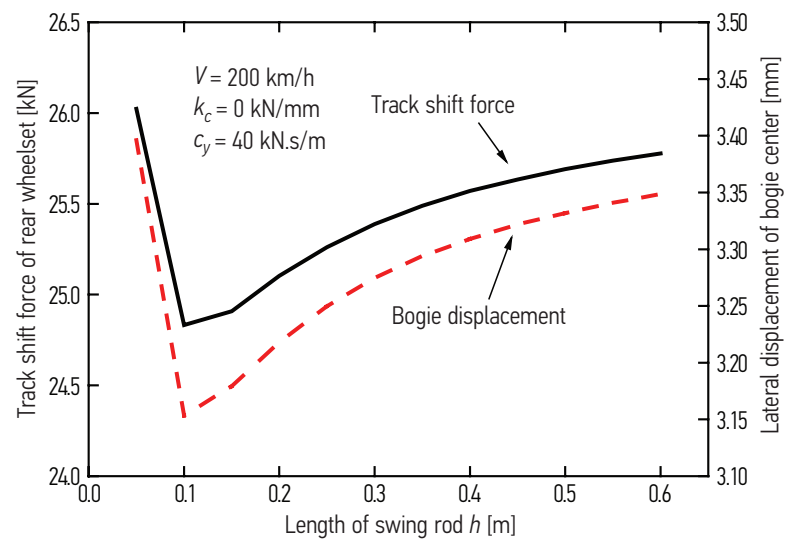

Fig. 21. The simulation results of locomotive 1 when the $k_{c}$ is $0 \mathrm{kN} / \mathrm{mm}$

the $k_{c}$ is 0 or $0.43 \mathrm{kN} / \mathrm{mm}$. This is the same as the demonstration in Fig. 18. The maximum track shift forces and displacement of the bogie are $48 \mathrm{kN}$ and $9.3 \mathrm{~mm}$ respectively when the length of the swing rod is 0 , which means the connection between the drive system and the frame is non-elastic. The lateral force of locomotive 1 is reduced by $45 \%$ when the drive system is in flexible suspension compared to the non-elastic connection.

Simulation results of the different $c_{y}$ of locomotive 1 on the track shift forces and lateral displacements of the bogie frame and drive system are shown in Fig. 23. The track shift forces and the lateral displacement of the bogie frame are minimal when the $c_{y}$ equals 10 to 20 $\mathrm{kN} \cdot \mathrm{s} / \mathrm{m}$, while the higher $c_{y}$ is favorable for reducing the relative displacement between the drive system and bogie frame.

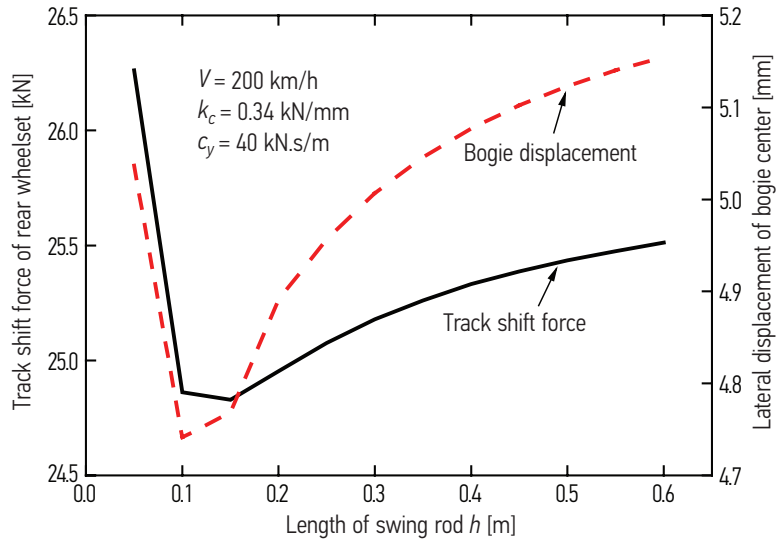

Fig. 22. The simulation results of locomotive 1 when the $k_{c}$ is $0.34 \mathrm{kN} / \mathrm{mm}$

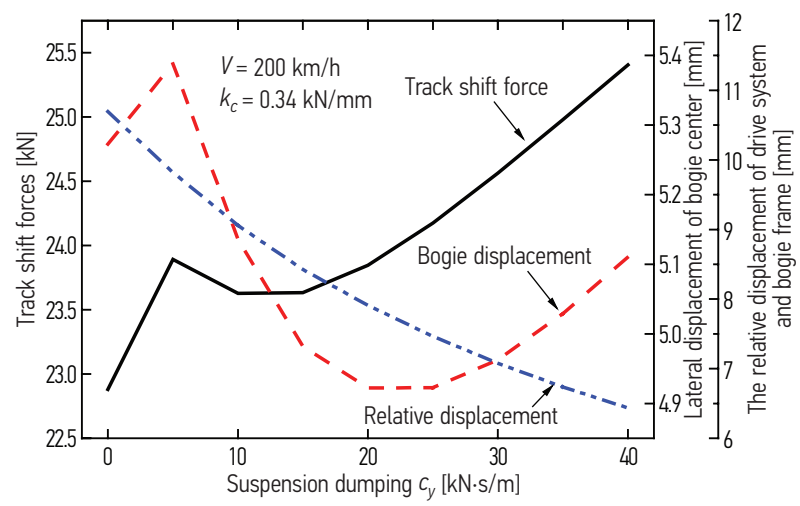

Fig. 23. The simulation results of locomotive 1 with different $c_{y}$

Figs 24 and 25 show the effect of differing swing rod lengths on the track shift forces of the locomotive 2 . The track shift forces and lateral displacement of the bogie are minimal when the length of swing rod is $0.1 \mathrm{~m}$. When the $k_{c}$ is $0.34 \mathrm{kN} / \mathrm{mm}$, the increasing length of the swing rod is better for dynamic vehicle performance, as is demonstrated in Fig. 9. The track shift force of locomotive 2 reduces $34 \%$ when the drive system is in flexible suspension compared to the non-elastic connection.

Simulation results of the different $c_{y}$ of locomotive 2 on the track shift forces and lateral displacements of the bogie frame and drive systems are shown in Fig. 26. The track shift forces and the lateral displacement of bogie frame are minimal when the $c_{y}$ equals 5 to $10 \mathrm{kN} \cdot \mathrm{s} / \mathrm{m}$, while the higher $c_{y}$ is favorable for reducing the relative displacement between the drive system and bogie frame. 


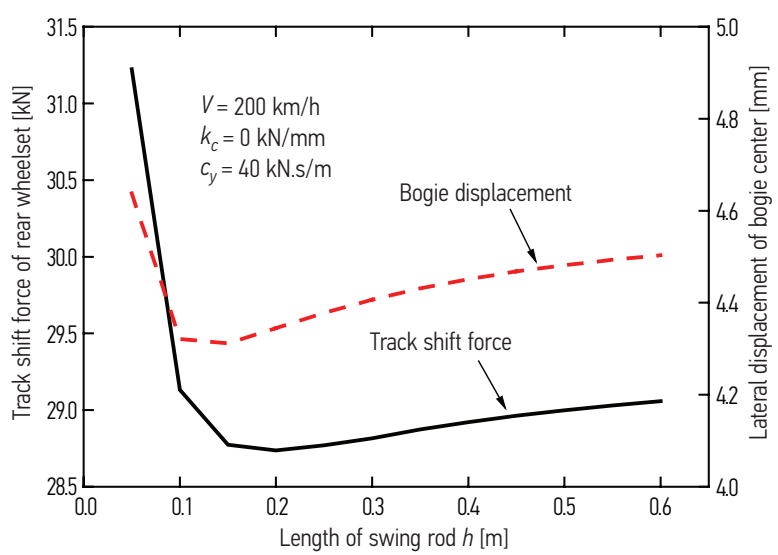

Fig. 24. The simulation results of locomotive 2 when the $k_{c}$ is $0 \mathrm{kN} / \mathrm{mm}$

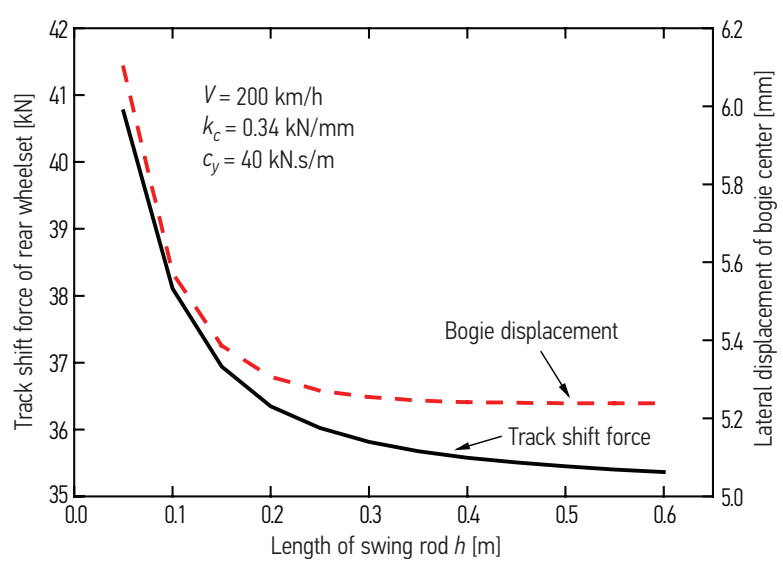

Fig. 25. The simulation results of locomotive 2 when the $k_{c}$ is $0.34 \mathrm{kN} / \mathrm{mm}$

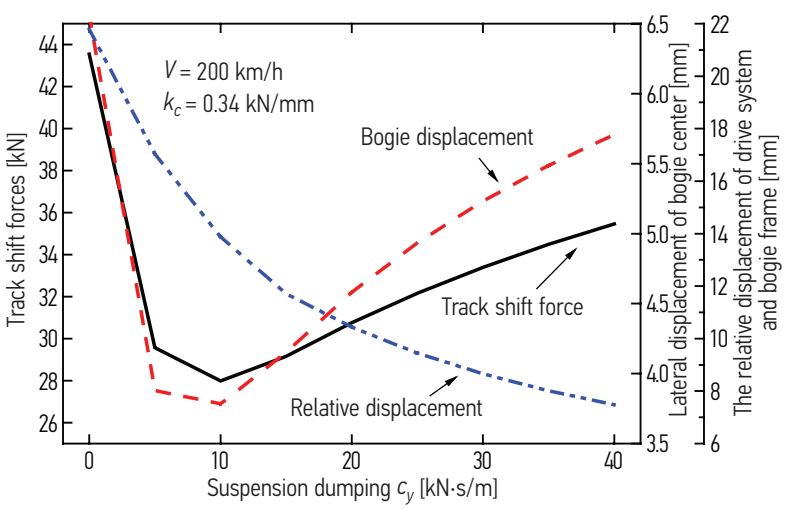

Fig. 26. The simulation results of locomotive 2 with different $c_{y}$

The nonlinear critical speeds of the locomotives are calculated according to the EN14363 (Ploach 2006; Yao et al. 2011). The optimal lengths of the swing rod for locomotives 1 and 2 whose drive systems are suspended flexibly are different. Variations in critical speed with the changes in length of the swing rod are shown in Fig. 27.

There is no optimal swing rod length for any drive system due to the changes in hunting frequencies and the changes of the running speeds and wheel conicity. The vehicle dynamics analysis shows that when the

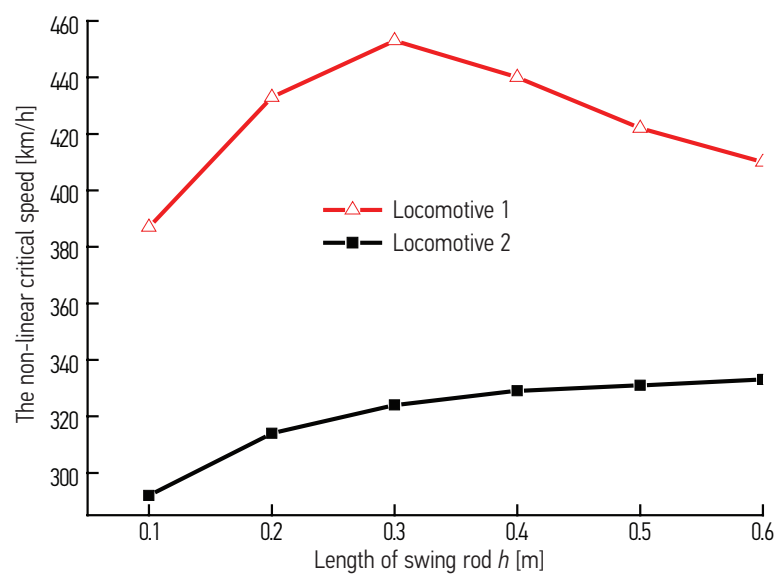

Fig. 27. The effect of length of swing rod on the non-linear critical speed

length of the swing rod is longer than $0.1 \mathrm{~m}$, the suspension frequency of the drive system is lower than $3 \mathrm{~Hz}$. Therefore, the flexible suspension of the drive systems has an obvious role in enhancing the vehicle dynamics performances, while no apparent influence can be made by further increasing the length of swing rod.

\section{Conclusions}

1. The drive system mounted on the bogie frame flexibly will help to improve locomotive dynamic performances. When the suspension frequency of the drive system is close to the wheelset hunting, the dynamic performance will be the best. The relative movement of the drive system to the bogie frame absorbs the movement energy of the frame that reduces the amplitude of hunting on the frame, which improves the lateral stability and reduces the track shift forces of locomotives.

2. The suspension frequency of the drive system depends on the length of the hanging rods, and the lateral synthesis stiffness of the double hollow shaft transmission with the corresponding position parameters. When the swing rods and the transmission are located on the same side, the effect of the lateral synthesis stiffness due to the double hollow shaft transmission equipment on the suspension frequency is obvious. When the length of the swing rods is greater than $100 \mathrm{~mm}$, the suspension frequency of the drive system is less than $3 \mathrm{~Hz}$.

3. When the swing rods and transmission equipment are located on the same side, the improvement of the dynamic performance of the locomotive is more visible. The axle lateral forces of locomotives whose hanging rods are located on the motor side and non-motor side are decreased by $45 \%$ and $34 \%$ respectively, relative to the non-flexible suspension of the drive system.

4. When the length of the swing rods of the front and rear drive systems are different in the same bogie, the locomotive dynamic performances are better within a broader range of speeds and the slopes of the tread.

5. The suspension frequency of the drive system should be close to the hunting frequency of the wheelset. 
The appropriate suspension damping of the drive system is conducive to improve the dynamic performance of the locomotive, but in order to reduce the relative lateral displacement of the drive system to the bogie frame, it is necessary to increase the suspension damping.

\section{Acknowledgements}

Authors wish to acknowledge the support of the $\mathrm{Na}$ tional Natural Science Foundation of China (No. 51205324, 51375043), the Fundamental Research Funds for the Central Universities (No. 2682014CX040) and Independent Research and Development Project of Key Laboratory (No. 2011TPL_T04).

\section{References}

Alfi, S.; Mazzola, L.; Bruni, S. 2008. Effect of motor connection on the critical speed of high-speed railway vehicles, Vehicle System Dynamics 46(Supplement 1): 201-214. http://dx.doi.org/10.1080/00423110801935814

Hödl, H.; Haigermoser, A. 1992. Entwicklung eines modernen Triebdrehgestellkonzepts für Hochleistungslokomotiven, Zeitschrift für Eisenbahnwesen und Verkehrstechnik 116(89): 344-353 (in German).

Lata, M. 2008. The modern wheelset drive system and possibilities of modelling the torsion dynamics, Transport 23(2): 172-181. http://dx.doi.org/10.3846/1648-4142.2008.23.172-181

Luo, Y. 2005. Study on Suspension Constructs and Parameters of Driving Equipment in Locomotives: $\mathrm{PhD}$ thesis. Southwest Jiaotong University (in Chinese).

Luo, Y; Jin, D. 2007. Research on the rules of suspension parameters to driving equipments suspended in bogie frames, China Railway Science 28: 78-82 (in Chinese).

Luo, Y; Luo, S. H.; Jin, D.-C. 2005. Research on driving equipment suspension parameters and the structures of locomotives with motor elastically mounted on the frame, China Railway Science 26: 57-61 (in Chinese).

Middendorf, E. 1996. Die neue Generation elektrischer Lokomotiven für die Deutsche Bahn AG: Die Baureihen 101, 145 und 152, ETR - Eisenbahntechnische Rundschau 45(6): 349-356 (in German).

Ploach, O. 2006. On non-linear methods of bogie stability assessment using computer simulations, Proceedings of the Institution of Mechanical Engineers, Part F: Journal of Rail and Rapid Transit 220(1): 13-27. http://dx.doi.org/10.1243/095440905X33251

Segieth, C. 1996. La 12X: Locomotive modulaire à grande puissance, Rail International 27(7): 24-30 (in French).

Sun, Z.-C. 1993. The application of anti-resonance theory in design of vibration absorbers, Chinese Journal of Applied Mechanics 4(10): 97-103 (in Chinese).

Yao, Y.; Zhang, H.-J.; Li, Y.-M.; Luo, S.-H. 2011. The dynamic study of locomotives under saturated adhesion, Vehicle System Dynamics 49(8): 1321-1338. http://dx.doi.org/10.1080/00423111003668195

Zhang, H.-J.; Chen, X.-H.; Sun, Y.-P.; Luo, Y.; Zhong, W.-S. 2007. The analysis of bogie platform of $200 \mathrm{~km} / \mathrm{h}$ highspeed passenger locomotive in our country, Journal of the China Railway Society 29: 101-106 (in Chinese).
Zhang, H.-J.; Huang, Y.-M.; Li, G.-J.; Luo, Y.; Zhong, W.-S. 2008. Research on the speed-increase six-axle locomotive with driving equipment flexibly suspended on the frame, China Railway Science 29: 84-88 (in Chinese). 